\title{
Dispersão larval radial pós-alimentar em Lucilia cuprina (Diptera, Calliphoridae): profundidade, peso e distância de enterramento para pupação
}

\author{
Leonardo Gomes ${ }^{1} \&$ Claudio José Von Zuben ${ }^{1,2}$ \\ 1. Depto de Zoologia, Universidade Estadual Paulista, Av. 24A, 1515, 13506-900 Rio Claro, SP, Brasil. (leugomes@ yahoo.com.br) \\ 2. Bolsista do CNPq.
}

\begin{abstract}
Postfeeding radial larval dispersion in Lucilia cuprina (Diptera, Calliphoridae): depth, weight and distance of burying for pupation. Blowflies utilize discrete and ephemeral sites for breeding and larval nutrition. After the exhaustion of food, the larvae begin dispersing to search for sites to pupate or for additional food source, process referred as postfeeding larval dispersal. Some aspects of this process were investigated in Lucilia cuprina (Wiedemann, 1830), utilizing a circular arena to permit the radial dispersion of larvae from the food source in the center. To determine the localization of each pupa, the arena was split into 72 equal sectors from the center. For each pupa, distance from the center of arena, weight and depth were determined. Statistical tests were performed to verify the relation among weight, depth and distance of burying for pupation. It was verified that the larvae that disperse farthest are those with lowest weights. The majority of individuals reached the depth of burying for pupation between 7 and $18 \mathrm{~cm}$. The study of this process of dispersion can be utilized in the estimation of postmortem interval (PMI) for human corpses in medico-criminal investigations.
\end{abstract}

KEYWORDS. Blowflies, Calliphoridae, Lucilia, forensic entomology, larval dispersal.

\section{INTRODUÇÃO}

As moscas-varejeiras utilizam-se de substratos discretos e efêmeros para posturas dos ovos e para alimentação das larvas (HANSKI, 1987). O estágio larval é o principal período em que ocorre limitação de recursos alimentares, e a competição por esses recursos é, geralmente, do tipo exploratório (Reis et al., 1994), em que cada larva procura ingerir o máximo de alimento possível, antes da completa exaustão dos recursos (UlLyetT, 1950). Após essa competição, as larvas começam a procurar um sítio para pupação, ou por mais fontes de alimento adicional, no caso daquelas larvas que não obtiveram o peso mínimo para a pupação. $\mathrm{O}$ processo é denominado dispersão larval pós-alimentar (GREENBERG, 1990).

Dentre as espécies de moscas-varejeiras, a cosmopolita Lucilia cuprina (Wiedemann, 1830) é uma das mais importantes do ponto de vista médico-veterinário, porque tanto na África como na Austrália, é responsável pela maioria dos casos relatados de míáases em ovinos (Zumpt, 1965; Guimarães et al., 1983; Norris, 1990; MARICONI et al., 1999). No Brasil, pelo fato de ser eussinantrópica e comumente encontrada em lixo urbano, substratos de carne em decomposição, frutos caídos, néctar de flores e fezes humanas, é importante como possível vetora de enteropatógenos para o homem (LiNHARES, 1981; Furlanetto et al., 1984; Almeida \& Mello, 1996; MARICONI et al., 1999). Os dípteros que se alimentam de matéria orgânica de origem animal em decomposição são de importância em entomologia forense por indicarem o tempo de decomposição de cadáveres humanos (WELLS \& GreEnBerg, 1992; Von Zuben et al., 1996). A ocorrência da introdução acidental de espécies exóticas de mos- cas-varejeiras do gênero Chrysomya (RubineauDesvoidy, 1830) no continente americano em meados do anos 70 (Guimarães et al., 1978), tem interessado aos ecólogos, no sentido de compreender o impacto dessa introdução sobre outras espécies de moscas-varejeiras, dentre elas L. cuprina.

A compreensão dos fenômenos biológicos envolvidos neste processo de introdução, tais como competição intra e interespecífica por recursos alimentares, substratos para oviposição e sítios para pupação para as moscas-varejeiras, depende da investigação de determinados parâmetros populacionais, além da estrutura ambiental espaço-temporal em que ocorrem estes fenômenos (Hengeveld, 1989). Além desses, ocorrem outros de pequena escala espacial como no caso de dispersão dos estágios imaturos dessas moscas.

O objetivo foi estudar sob condições experimentais, em simulação de ambiente natural, a dispersão larval radial pós-alimentar em L. cuprina e tentar estabelecer relação entre as variáveis peso, distância e profundidade de enterramento em uma arena que permitisse às larvas deslocamento em todas as direções.

\section{MATERIAL E MÉTODOS}

Adultos de L. cuprina foram coletados no Instituto de Biociências, Universidade Estadual Júlio de Mesquita Filho (UNESP), em Rio Claro, SP, utilizando como isca carcaça de peixe em decomposição. Os exemplares foram identificados e mantidos em gaiolas teladas, em sala com temperatura de $25 \pm 1{ }^{\circ} \mathrm{C}, 60 \%$ de umidade relativa e fotofase de 12 horas, sendo ministrados água, açúcar e fígado bovino ad libitum. 
Uma arena circular foi montada sobre o piso da sala, iluminada de forma homogênea (a fim de evitar tendências na dispersão das larvas), forrada com serragem, tendo suas margens delimitadas por papelão e dividida em 72 setores de cinco graus cada. $O$ desenvolvimento larval da geração $\mathrm{F}_{2}$ deu-se em carne moída, substrato este colocado no centro da arena circular, para que as larvas abandonassem o mesmo e irradiassem para empupar. Foram montados para esse estudo da dispersão larval radial pós-alimentar, três experimentos em uma mesma arena com $50 \mathrm{~cm}$ de diâmetro e forrada com uma camada de $20 \mathrm{~cm}$ de serragem. Esta arena, com maior profundidade em relação a outros experimentos (Gomes et al., 2002), teve como objetivo verificar a profundidade máxima que as larvas se enterrariam.

As pupas foram localizadas e retiradas da serragem, sendo determinadas as suas localizações por setor, e estimados, para cada uma, a distância, a profundidade e o peso, com o auxílio de régua, trena e balança analítica. A coleta das pupas começou da periferia para o centro da arena. Cada pupa foi individualizada em frasco plástico, antes que ocorresse a emergência do adulto. Os indivíduos adultos foram sexados. O material testemunho foi depositado na coleção entomológica do Departamento de Zoologia da UNESP, Rio Claro. As variáveis estudadas foram avaliadas estatisticamente através de testes de regressão e igualdade de médias no nível de 0,05 de significância (ZAR, 1999).

\section{RESULTADOS}

Foram obtidos valores, nos três experimentos, para profundidade de enterramento, distância em relação ao centro da arena e peso, para um total de 783 pupas de $L$. cuprina (tab. I). A utilização de uma arena circular permitiu a locomoção das larvas em todas as direções, observando-se que pode haver maior concentração de larvas em alguns quadrantes desse substrato de pupação.
Verificou-se uma concentração maior das pupas (74) no terceiro quadrante (fig. 1). A profundidade média de enterramento foi de $14,58 \mathrm{~cm}$, considerando os resultados dos três experimentos conjuntamente, mas nota-se que há uma concentração, em média, de quase 45 larvas que se enterraram a $20 \mathrm{~cm}$ (fig. 2). Quando se considera a distribuição das pupas por distância média de enterramento, pode-se notar que a maioria delas se concentrou entre 15 e $20 \mathrm{~cm}$ (fig. 3), sendo que 12 larvas atingiram a distância máxima possível durante a dispersão.

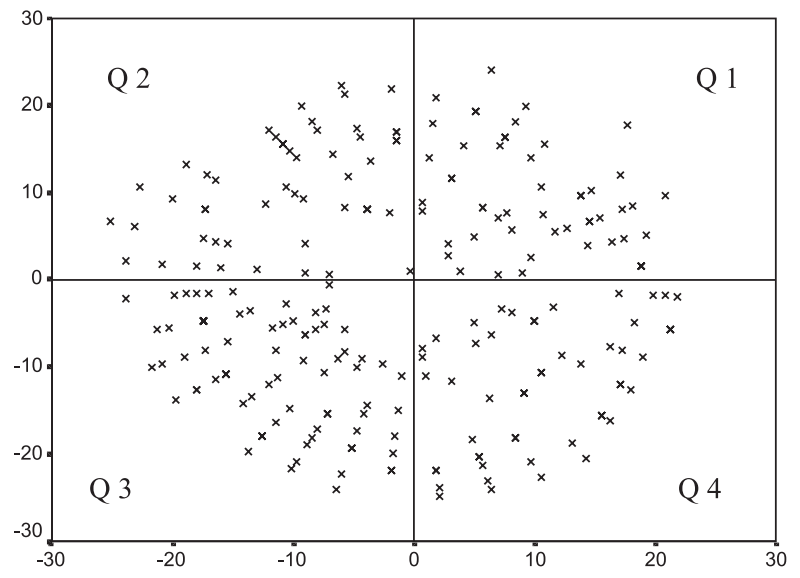

Fig. 1. Distribuição das pupas de Lucilia cuprina na arena (quadrante 1 superior à direita, demais na seqüência no sentido anti-horário). Cada ponto pode representar mais de uma pupa.

Quanto à distribuição média das pupas por peso (mg), observa-se que 77 pupas atingiram o peso entre 18 e $21 \mathrm{mg}$ e apenas 4 pupas, $31 \mathrm{mg}$ (fig. 4). Isto provavelmente deve-se ao fato que alguns poucos indivíduos de maior peso caminharam mais do que a maioria desta faixa de peso. Estatisticamente a correlação entre peso e distância é negativa e significante (-0,386 a $5 \%$ ) e entre peso e profundidade não houve correlação significativa. Isso significa que as larvas mais leves geralmente foram as que percorreram maiores distâncias.

Tabela I. Valores de média (x) e desvio-padrão (DP) de profundidade (cm), distância (cm) e peso (mg) por sexo das pupas de Lucilia cuprina distribuídas em três experimentos para o estudo da dispersão larval.

\begin{tabular}{|c|c|c|c|c|}
\hline & Sexo & $\begin{array}{l}\text { Experimento } 1 \\
x \pm D P \\
(N)\end{array}$ & $\begin{array}{l}\text { Experimento } 2 \\
x \pm D P \\
(N)\end{array}$ & $\begin{array}{l}\text { Experimento } 3 \\
x \pm D P \\
(N)\end{array}$ \\
\hline \multirow[t]{4}{*}{ Profundidade } & Indeterminado & $11,90 \pm 5,58$ & $16,09 \pm 4,96(104)$ & $15,79 \pm 5,42(98)$ \\
\hline & Fêmea & $11,60 \pm 5,03(90)$ & $16,13 \pm 3,45(45)$ & $16,88 \pm 4,72(52)$ \\
\hline & Macho & $11,70 \pm 5,33(110)$ & $15,10 \pm 5,30$ & $15,64 \pm 5,19$ \\
\hline & Total & $11,70 \pm 5,23(230)$ & $15,63 \pm 4,99(282)$ & $15,93 \pm 5,19$ \\
\hline \multirow[t]{4}{*}{ Distância } & Indeterminado & $16,80 \pm 4,26(30)$ & $10,73 \pm 3,67(104)$ & $10,56 \pm 3,00(98)$ \\
\hline & Fêmea & $17,00 \pm 5,77(90)$ & $9,47 \pm 3,81(45)$ & $10,63 \pm 2,51(52)$ \\
\hline & Macho & $16,60 \pm 5,06(110)$ & $10,22 \pm 3,91$ & $10,18 \pm 3,26$ \\
\hline & Total & $16,80 \pm 5,24(230)$ & $10,27 \pm 3,82(282)$ & $10,41 \pm 3,03(271)$ \\
\hline \multirow[t]{4}{*}{ Peso } & Indeterminado & $18,70 \pm 3,21$ & $14,92 \pm 3,95(104)$ & $14,88 \pm 4,57(98)$ \\
\hline & Fêmea & $20,70 \pm 2,95(90)$ & $16,06 \pm 4,96(45)$ & $14,38 \pm 4,20(52)$ \\
\hline & Macho & $17,50 \pm 2,79(110)$ & $15,42 \pm 4,40(133)$ & $15,34 \pm 4,65(121)$ \\
\hline & Total & $18,90 \pm 3,26(230)$ & $15,34 \pm 4,29(282)$ & $15,99 \pm 4,54(271)$ \\
\hline
\end{tabular}




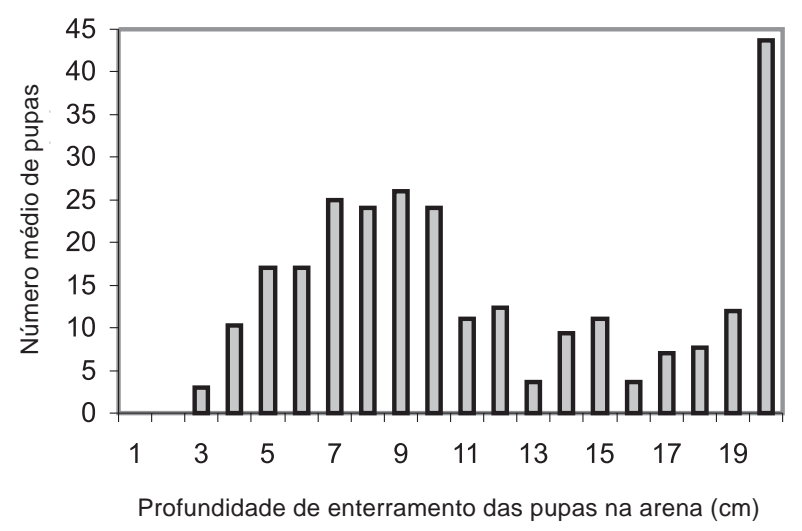

Fig. 2. Distribuição das pupas de Lucilia cuprina por profundidade de enterramento $(\mathrm{cm})$, considerando os três experimentos conjuntamente (ANOVA, P, 2,78 a $5 \%$ de significância).

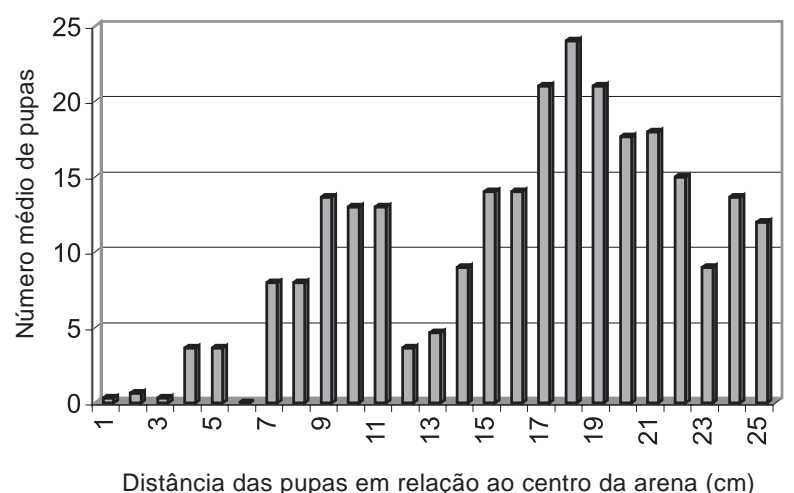

Fig. 3. Distribuição das pupas de Lucilia cuprina por distância de enterramento (cm), considerando os três experimentos conjuntamente (ANOVA, P, 2,78 a $5 \%$ de significância).

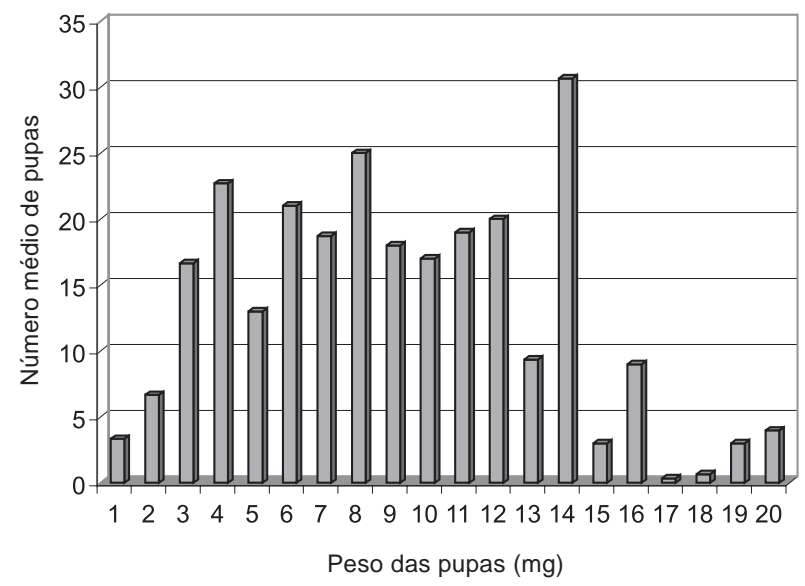

Fig. 4. Distribuição das pupas de Lucilia cuprina por peso (mg), considerando os três experimentos conjuntamente (ANOVA, P, 2,78 a $5 \%$ de significância).

\section{DISCUSSÃO}

Os primeiros estudos realizados no Brasil tinham considerado para o processo de dispersão larval, uma calha de $3 \mathrm{~m}$ de comprimento por $30 \mathrm{~cm}$ de largura que permitia a locomoção das larvas em apenas uma direção e dois sentidos (Godoy et al., 1995, 1996; BASSANEZI et al., 1997). Experimentos considerando uma arena circular refletem melhor as condições de ambiente natural que as larvas vão enfrentar ao abandonarem o substrato alimentar em busca de um sítio para pupação, porque permitem uma dispersão radial das larvas a partir do substrato alimentar localizado no centro, e não simplesmente uma dispersão numa única direção e apenas dois sentidos, como no caso de uma calha (Gomes et al., 2002).

O processo de dispersão de larvas de $L$. cuprina em uma arena circular confirma resultados anteriores com califorídeos do gênero Chrysomya (Gomes \& Von ZuBen, 2002) utilizando arenas com maior diâmetro e menor profundidade, em que as larvas com peso menor são as que têm tendência de se deslocar mais, talvez por estarem à procura de nova fonte alimentar e não estarem buscando distâncias maiores para se enterrarem para empupar (GoMES et al., 2002). No presente estudo, observou-se correlação negativa entre a distância percorrida para pupação e peso das pupas. Isto confere com o experimento de Gomes et al. (2002), onde o peso de larvas de C. megacephala (Fabricius, 1974) diminui com a distância percorrida para localização do sítio de pupação.

A maior concentração de pupas foi observada em profundidades superiores a $12 \mathrm{~cm}$ e em relação à distância, 12 larvas chegaram a atingir a distância máxima possível a partir do centro $(25 \mathrm{~cm})$. Aparentemente, o que mais limitou a movimentação das larvas foi a distância e não a profundidade, embora a utilização de uma arena com essas dimensões de $20 \mathrm{~cm}$ de profundidade e $50 \mathrm{~cm}$ de diâmetro tenha possibilitado profundidades maiores quando comparada a estudos anteriores (Gomes et al., 2002).

Godoy et al. $(1995,1996)$ observaram tanto para C. megacephala como para Chrysomya putoria (Wiedemann, 1830) uma oscilação na freqüência de pupas em função da distância a partir do substrato alimentar. Segundo BolDRINI et al. (1997), estas oscilações seriam uma conseqüência da formação de agregações larvais em determinado local do substrato de pupação. Foi sugerido que as larvas dispersantes seriam capazes de perceber a densidade de larvas já enterradas em um determinado ponto do substrato, sendo que aglomerações de larvas num determinado ponto induziriam aquelas que ainda estavam caminhando a procurar sítios de pupação mais distantes. No presente estudo, o fato de algumas larvas alcançarem pontos muito distantes do centro da arena pode estar relacionado à esta percepção de larvas já enterradas ou ainda associado à busca por novo substrato alimentar para terminar seu ciclo de desenvolvimento.

O comportamento de dispersão larval pós-alimentar e o conseqüente padrão de distribuição espacial das pupas nos sítios de pupação podem ter implicações na maior ou menor suscetibilidade das mesmas ao ataque de predadores e parasitóides, em populações de ambientes naturais (LEGNER, 1977). Em ambiente natural, as larvas de $L$. cuprina e de outras espécies de califorídeos, como C. megacephala, podem atingir profundidades e distâncias variadas para pupação, dependendo do tipo de substrato e nível de compactação do mesmo a partir do centro de dispersão (GOMES \& Von ZuBEn, 2002).

Os estudos de dispersão podem ter importantes 
implicações para investigações médico-legais, porque a presença de larvas e pupas em cadáveres humanos ou nas circunvizinhanças pode auxiliar na estimativa do intervalo de tempo entre a morte e a descoberta do cadáver, referido como intervalo pós-morte (IPM) (VoN ZuBEN et al., 1996). Essa estimativa constitui-se em aspecto fundamental em estudos de medicina legal (SмітH, 1986), sendo que a mesma pode ser seriamente prejudicada, levando a uma subestimativa do IPM, caso não sejam consideradas as larvas dispersantes (VON ZuBEN et al., 1996).

Agradecimento. À FAPESP pelo auxílio financeiro (Processos 98/09939-6 e 00/06336-0).

\section{REFERÊNCIAS BIBLIOGRÁFICAS}

Almeida, J. M. D’ \& Mello, R. P. 1996. Comportamento de dípteros muscóides frente a substratos de ovoposição, em laboratório, no Rio de Janeiro, RJ, Brasil. Memórias do Instituto Oswaldo Cruz, Rio de Janeiro, 91(1):131-136.

Bassanezi, R. C.; Leite, M. B. F. et al. 1997. Diffusion model applied to postfeeding larval dispersion in blowflies (Diptera: Calliphoridae). Memórias do Instituto Oswaldo Cruz, Rio de Janeiro, 92:281-286.

BoldRini, J. L.; Bassanezi, R. C. et al. 1997. Non-local interactions and the dynamics of dispersal in immature insects. Journal of Theoretical Biology, London, 185:523-531.

Furlanetto, S. M. P.; Campos, M. L. et al. 1984. Microorganismos enteropatogênicos em moscas africanas pertencentes ao gênero Chrysomya (Diptera: Calliphoridae) no Brasil. Revista de Microbiologia, São Paulo, 15:170-174.

Godoy, W. A. C.; Fowler, H. G. et al. 1995. Larval dispersion in Chrysomya megacephala, C. putoria and Cochliomyia macellaria (Diptera: Calliphoridae). Journal of Applied Entomology, Berlin, 119:263-266.

Godoy, W. A. C.; Reis, S. F. \& Von Zuben, C. J. 1996. Larval dispersal in Chrysomya megacephala, Chrysomya putoria and Cochliomyia macellaria (Diptera: Calliphoridae): ecological implications of aggregation behavior. Journal of Applied Entomology, Berlin, 120:423-426.

Gomes, L. \& Von Zuben, C. J. 2002. Dispersão larval pósalimentar em Chrysomya megacephala (F.) (Diptera: Calliphoridae): profundidade, distância e peso de enterramento para pupariação. Bioscience Journal, Uberlândia, 18:6776.

Gomes, L.; Von Zuben, C. J. \& Govone, J. S. 2002. Comportamento da dispersão larval radial pós-alimentar em moscas-varejeiras do gênero Chrysomya (Diptera: Calliphoridae): busca por novas fontes de alimento. Entomologia y Vectores, Rio de Janeiro, 9:115-132.
Greenberg, B. 1990. Behavior of postfeeding larvae of some Calliphoridae and a muscid (Diptera). Annals of Entomological Society of America, Maryland, 83:12101214.

Guimarães, J. H.; Papavero, N. \& Prado, A. N. 1983. As miíases na Região Neotropical (identificação, biologia, bibliografia). Revista Brasileira de Zoologia, São Paulo, 1(1):239-416.

Guimarães, J. H.; Prado, A. P. \& Linhares, A. X. 1978. Three newly introduced blowfly species in southern Brazil (Diptera: Calliphoridae). Revista Brasileira de Entomologia, São Paulo, 22(1):53-60.

HANSKI, I. 1987. Carrion fly community dynamics: patchness, seasonality and coexistence. Ecological Entomology, Oxford, 12:257-266.

Hengeveld, R. 1989. Dynamics of biological invasions. New York, Chapman \& Hall. 160p.

LEGNER, E. F. 1977. Temperature, humidity and depth of habitat influencing host destruction and fecundity of muscoid fly parasites. Entomophaga, Paris, 22:199-206.

Linhares, A. X. 1981. Synanthropy of Calliphoridae and Sarcophagidae (Diptera) in the city of Campinas, São Paulo, Brazil. Revista Brasileira de Entomologia, São Paulo, 25(1):189-215.

Mariconi, F. A. M.; Guimarães, J. H. \& Filho, E. B. 1999. A mosca doméstica e algumas outras moscas nocivas. Piracicaba, FEALQ. 135p

Norris, K. R. 1990. Evidence for the multiple exotic origin of Australia populations of sheep blowfly, Lucilia cuprina (Wiedemann) (Diptera; Calliphoridae). Australian Journal of Zoology, Sidney, 38:635-648.

Reis, S. F.; Stangenhaus, G. et al. 1994. Variação em caracteres bionômicos em função de densidade larval em Chrysomya megacepala e Chrysomya putoria (Diptera: Calliphoridae). Revista Brasileira de Entomologia, São Paulo, 38(1):33, 34.

Sмiтн, K. G. V. 1986. A manual of forensic entomology. Ithaca, Cornell University. 475p.

Ullyett, G. C. 1950. Competition for food and allied phenomena in sheep-blowfly populations. Phillip Transactions of Real Society of London, London, 234:77-174.

Von Zuben, C. J.; Bassanezi, R. C. et al. 1996. Theoretical approaches to forensic entomology: I. mathematical model of postfeeding larval dispersal. Journal of Applied Entomology, Berlin, 120:379-382.

Wells, J. D. \& Greenberg, B. 1992. Interaction between Chrysomya rufifacies and Cochliomyia macellaria (Diptera: Calliphoridae): the possible consequences of an invasion. Bulletin of Entomological Research, Walingford, 82:133137.

ZAR, J. H. 1999. Biostatistical analysis. New Jersey, Prentice Hall. 666p.

Zumpt, F. 1965. Myiasis in man and animals in the Old World. London, Butterworths. 267p. 